\title{
ECLETICA
}

www.scielo.br/eg

Volume 29, número 1, 2004

\section{Determinação de ultratraços de selênio em urina por geração de hidretos e espectrometria de absorção atômica em fluxo.}

\author{
N. M. M. Coelhol, N. Baccan' \\ ${ }^{1}$ Grupo de Espectroscopia Aplicada - Instituto de Quimica - Universidade Federal Uberlândia, CEP 38.400.902 - Uberlândia - \\ $M G$ - Brasil \\ ${ }^{2}$ Instituto de Quimica - Universidade Estadual de Campinas - CEP 13.083.970 - Campinas - SP - Brasil
}

Resumo: A determinação de selênio utilizando a geração de hidretos acoplada às técnicas atômicas vêm sendo utilizada com sucesso em amostras de urina. Devido a necessidade de se dispor de um método analítico capaz de determinar pequenas quantidades de selênio; é proposto o sistema de fluxo, por zonas coalescentes, utilizando a técnica de geração de hidretos, seguida da detecção por espectrometria de absorção atômica para a determinação de selênio em amostras de urina. O limite de detecção obtido é 0,3 $\mathrm{ng} \mathrm{mL}{ }^{-1}$, correspondendo a $30 \mathrm{pg}$ de selênio e a freqüência analítica de 140 determinações por hora. A precisão (d.p.r.) do método foi de $2,0 \%$ para $1,0 \mathrm{ng} \mathrm{mL}^{-1}$. A quantidade de reagente $\left(0,5 \mathrm{mg} \mathrm{de} \mathrm{NaBH}_{4}\right.$ para cada determinação) e de resíduo químico gerado no laboratório $\left(170 \mathrm{~mL} \mathrm{~h}^{-1}\right)$ é mínima. A aplicação do método proposto para análises de amostras de urina evidenciou a presença média de $4,0 \mathrm{ng} \mathrm{mL}^{-1} \mathrm{de}$ selênio para pacientes que não ingeriram nenhum medicamento contendo selênio. Para pacientes que ingeriram uma dose única de medicamento contendo selênio, o teor encontrado nas amostras de urina analisadas variaram de 5,0 a 16,0 $\mathrm{ng} \mathrm{mL}^{-1}$. Estudos de recuperação mostraram valores de 90,0\% e 103,0\% para 1,0 e 10,0 ng mL $\mathrm{nL}^{-1}$ de selênio adicionado, respectivamente, nas amostras de urina. A certificação do método foi avaliada para análises de material de referência de urina SRM 2670 (valor certificado: 30,0 0 8,0 ng $\mathrm{mL}^{-1} \mathrm{e}$ valor obtido: $27,0 \pm 2,0 \mathrm{ng} \mathrm{mL}^{-1}$ ).

Palavras-chave: selênio, ultratraços, injeção em fluxo, geração de hidretos, urina.

\section{Introdução}

O selênio é um elemento essencial para o homem e seu estudo é de grande interesse devido suas propriedades anti-oxidantes e anticancerígenas [11]. No entanto, este elemento apresenta um pequeno intervalo de concentração entre o nível essencial e o toxicológico, onde doses tóxicas são somente 100 vezes maiores do que aquelas necessárias para as funções fisiológicas.

O conteúdo de selênio na urina varia de acordo com a dieta alimentar. A concentração de selênio [1] na urina é de aproximadamente $30 \mathrm{mg} \mathrm{L}^{-1}$, no entanto, valores maiores podem ser encontrados em populações que vivem em áreas de solos seleníferos. Desde que a principal rota para a excreção de selênio no organismo humano é a urina, o conteúdo deste elemento é um indicador de casos de intoxicação e/ou deficiência de selênio no homem.

Os problemas analíticos apresentados para a determinação de selênio em urina são o alto conteúdo de sais e a presença de vários constituintes orgânicos encontrados nestas amostras [7].

O desenvolvimento de métodos analíticos para a determinação exata de baixos níveis de selênio é importante. O selênio pode ser determinado em amostras de urina por espectrometria de

(Autor para correspondência: e-mail: nmmcoelho@ufu.br, FAX:34-3239-4208) 
absorção atômica por forno de grafite [10] e por geração de hidretos [12], por espectrometria de fluorescência atômica [13] e molecular [9] e por espectrometria de massa com plasma indutivamente acoplado [8].

A espectrometria de absorção atômica quando acoplada à geração de hidretos oferece uma alternativa simples e sensível para a determinação de elementos que geram hidretos $[5,6]$ e portanto, esta técnica pode ser aplicada na determinação de selênio em diferentes tipos de amostras.

Sob este aspecto, a espectrometria de absorção atômica e geração de hidretos aliada aos sistemas de análise por injeção em fluxo é uma proposta para a automatização das etapas analíticas.

O principal objetivo do presente estudo foi desenvolver um procedimento simples e sensível para determinação de selênio em amostras de urina em ordem de validar uma metodologia para controle analítico em amostras de interesse clínico. O método consiste na introdução simultânea da amostra contendo selênio e do reagente redutor (borohidreto de sódio) em soluções transpor- tadoras contendo ácido clorídrico e água desionizada, respectivamente. $\mathrm{O}$ hidreto de selênio formado é separado num separador gás-líquido, e então, conduzido por nitrogênio para a cela de atomização. As principais vantagens apresentadas por esta técnica são: separação da espécie analítica da matriz, alta eficiência de introdução da amostra resultando em boa sensibilidade e fácil automatização. Adicionalmente, os resultados obtidos no presente estudo fornecem dados preliminares da presença deste elemento em amostras de urina e abre possibilidades para estudos relatando a presença de selênio em amostras de interesse clínico e interações na absorção, atividades biológicas e toxicidade.

\section{Procedimento Experimental}

\section{Instrumentação}

O sistema de fluxo empregado na determinação de selênio está representado na Figura 1.

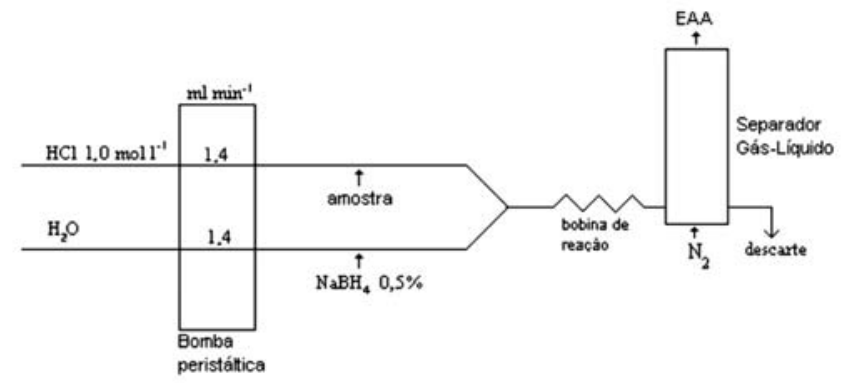

Figura 1. Sistema de fluxo proposto para determinação de selênio. E.A.A.: Espectrofotômetro de Absorção Atômica.

É utilizado o sistema de zonas coalescentes em confluência que consiste em introdução simultânea de volumes iguais de amostra e de reagente redutor, em fluxos de carregadores separados de mesma vazão. Neste caso, $100 \mathrm{~mL}$ de amostra e de reagente redutor $\left(\mathrm{NaBH}_{4} 0,5 \%(\mathrm{~m} / \mathrm{v})\right)$ são introduzidos em linhas transportadoras de ácido clorídrico $\left(1,0 \mathrm{~mol} \mathrm{~L}^{-1}\right)$ e água desionizada com vazões de 1,4 mL min'-1, respectivamente. As soluções são impulsionadas por uma bomba peristáltica Gilson, modelo Minipuls 3 com 8 canais, utilizando tubos de tygon para o bombeamento. Tubos de polietileno de 1,0 mm de diâmetro interno são usados para a construção do sistema de fluxo. A unidade de comutação para a introdução das amostras é um injetor tipo proporcional [2]. Para a separação do hidreto formado é utilizado um separador gás-líquido do tipo varredura [3]. O hidreto de selênio gerado é separado da fase líquida no separador gáslíquido, sendo transportado ao atomizador usando nitrogênio ultra-puro como gás de arraste com vazão controlada por um regulador de vazão de gás. 
O atomizador eletrotérmico é tipo "T" com a câmara de atomização de $17 \mathrm{~cm}$ de comprimento e $0,8 \mathrm{~cm}$ de diâmetro interno [4]. O atomizador é enrolado com 2,5 metros de fio Ni-Cr e mantém a temperatura adequada para a atomização do hidreto de selênio através da resistência de $\mathrm{Ni}$-Cr conectada a um regulador de voltagem, Varivolt. As medidas de absorbância foram feitas em um Espectrofotômetro de Absorção Atômica, Intralab, modelo Gemini AA12/1475, equipado com lâmpada de cátodo oco de selênio e corretor de fundo (lâmpada de deutério) ligado para corrigir o sinal analítico. As leituras de absorbância foram feitas em $196 \mathrm{~nm}$ com abertura de fenda de $0,5 \mathrm{~nm}$ e corrente de lâmpada de $10 \mathrm{~mA}$.

\section{Reagentes e padrões}

As soluções utilizadas neste estudo foram preparadas usando água desionizada (resistividade $18,2 \mathrm{MWcm}$ ) e reagentes de grau analítico, usando balões volumétricos para o acerto de volumes finais. Frascos de polietileno foram empregados na estocagem de soluções.

Solução padrão de selênio: preparada a partir de 1,4040 g de dióxido de selênio $\left(\mathrm{SeO}_{2}\right.$ - Riedel) em 1 litro de água. As soluções padrão de selênio, de concentrações variáveis entre 1,0 a $100 \mathrm{ng} \mathrm{mL}$ ${ }^{1}$ foram obtidas por diluição adequada de uma solução estoque de $10,0 \mathrm{mg} \mathrm{mL}^{-1}$ de selênio, com solução de $\mathrm{HCl} 1,0 \mathrm{~mol} \mathrm{\textrm {L } ^ { - 1 }}$. Estas diluições foram feitas empregando-se microbureta de vidro.

Solução de borohidreto de sódio $0,50 \%(\mathrm{~m} / \mathrm{v})$ : preparada por dissolução de $0,50 \mathrm{~g} \mathrm{de} \mathrm{NaBH}_{4}$ (Merck) em 100,0 mL de $\mathrm{KOH}$ (Merck) 0,05 $\mathrm{mol} \mathrm{L}^{-1}$.

Solução de ácido clorídrico $1,0 \mathrm{~mol} \mathrm{~L}^{-1}$ : preparada a partir de $83,3 \mathrm{~mL}$ de $\mathrm{HCl}$ concentrado (Merck) por diluição a 1 litro de água desionizada.

\section{Material de referência certificado (SRM 2670)}

As amostras de urina de material certificado SRM 2670 (nível normal e nível elevado de selênio) foram reconstituídas, adicionando $20,0 \mathrm{~mL}$ de água desionizada em cada frasco. Em alíquotas de 10,0 mL de urina para cada nível de selênio foram adicionados $10,0 \mathrm{~mL}$ de $\mathrm{HNO}_{3}$ concentrado. As amostras foram aquecidas em chapa de aqueci- mento até quase a secura, quando foram adicionados 3,0 mL de $\mathrm{HCl}$ concentrado e, então evaporadas novamente até quase secar. A seguir, o volume foi completado com água desionizada a $10,0 \mathrm{~mL}$ com $\mathrm{HCl}$ 1,0 mol L-1 para a amostra de urina de nível normal de selênio e $25,0 \mathrm{~mL}$ para a amostra de urina de nível elevado de selênio.

\section{Amostras de urina com adição de selênio}

As amostras de urina de pacientes que não estavam sob administração de medicamentos contendo selênio, foram coletadas e, em seguida, foram adicionadas quantidades conhecidas de selênio $(1,0 \mathrm{ng}$ $\mathrm{mL}^{-1}$ e $10,0 \mathrm{ng} \mathrm{mL}^{-1}$ ) e $10,0 \mathrm{~mL}$ de $\mathrm{HNO}_{3}$ concentrado. As amostras foram aquecidas em chapa de aquecimento até quase a secura, quando foram adicionados 3,0 $\mathrm{mL}$ de $\mathrm{HCl}$ concentrado e, então, evaporadas novamente até quase secar. A seguir, o volume foi completado a 25,0 mL com $\mathrm{HCl} 1,0 \mathrm{~mol} \mathrm{~L}^{-1}$.

\section{Amostras de urina de pacientes submetidos a uma} dose única de medicamento contendo selênio

Os pacientes submetidos ao estudo ingeriram uma dose única de medicamento. Conforme dados do fabricante, a composição do medicamento é de $50 \mathrm{mg}$ de selênio na forma de complexo por $350 \mathrm{mg}$ de medicamento. As amostras de urina foram coletadas nos seguintes tempos: 0 hora, isto é, antes da ingestão do medicamento e após ingestão do medicamento nos seguintes tempos: $6,12,18$, $24,36,48,60$ e 72 horas. Às amostras foram adicionados $10,0 \mathrm{~mL}$ de $\mathrm{HNO}_{3}$ concentrado e, então aquecidas em chapa de aquecimento até quase secura. Em seguida, foram adicionados $3,0 \mathrm{~mL}$ de $\mathrm{HCl}$ concentrado e, novamente, foram evaporadas até quase secura. A seguir, o volume foi completado a $25,0 \mathrm{~mL}$ com $\mathrm{HCl} 1,0 \mathrm{~mol} \mathrm{~L}^{-1}$.

\section{Resultados e discussão}

Avaliação das condições experimentais para determinação de selênio

As condições experimentais para determinação de selênio foram estabelecidas empregando o sistema de fluxo esquematizado na Figura 1. Os 
seguintes parâmetros foram avaliados: bobina de reação, volume injetado, concentração de $\mathrm{NaBH}_{4}$ e de $\mathrm{HCl}$, vazão das soluções transportadoras, vazão do gás de arraste e temperatura de atomização. Soluções padrão de selênio de 30, 60 e $90 \mathrm{ng} \mathrm{mL}^{-1}$ foram utilizadas para estes estudos.

A eficiência da geração do hidreto de selênio foi avaliada utilizando bobinas de reação de diferentes tamanhos $(10,20,30,50$ e $80 \mathrm{~cm})$. A Figura 2 mostra o efeito do comprimento da bobina de reação para um volume de $100 \mathrm{~mL}$ de selênio e de borohidreto de sódio. O sinal analítico é maior para a bobina de $20 \mathrm{~cm}$, diminuindo quando o tempo de reação aumentou, devido à dispersão sofrida pela amostra no percurso analítico. A bobina de $20 \mathrm{~cm}$ foi usada para a continuidade deste trabalho.

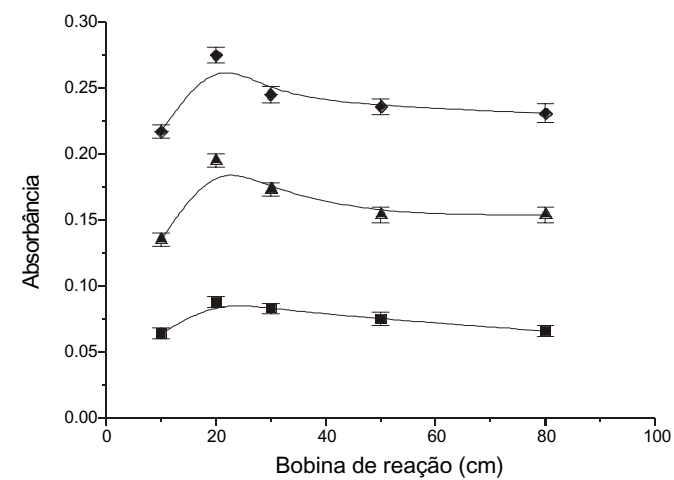

Figura 2. Efeito do comprimento da bobina de reação na determinação de selênio.

Condições experimentais: concentração de $\mathrm{NaBH}_{4}: 1,0 \%$ (m/ v), concentração de $\mathrm{HCl}: 1,0 \mathrm{~mol} \mathrm{~L}^{-1}$, volume injetado: $100 \mathrm{~mL}$, vazão dos carregadores: $1,5 \mathrm{~mL} \mathrm{~min}^{-1}$, vazão de nitrogênio: 150

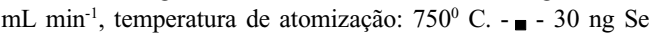
$\mathrm{mL}^{-1},-\mathbf{\iota}^{-} 60 \mathrm{ng}$ Se $\mathrm{mL}^{-1},-\bullet-90 \mathrm{ng} \mathrm{Se} \mathrm{mL}^{-1}$.

No sistema de fluxo proposto, a amostra e a solução de $\mathrm{NaBH}_{4}$ são injetadas em soluções transportadoras de $\mathrm{HCl}$ e água, respectivamente. A influência de volumes injetados de amostra e de redutor na eficiência da formação do hidreto de selênio foi avaliada na faixa de 50-200 mL. A Figura 3 mostra que o sinal de absorbância aumentou com o volume injetado de amostra e de redu- tor, sendo que o sinal foi maior para $150 \mathrm{~mL}$, mantendo-se constante para volumes maiores. Neste caso, para volumes maiores que $100 \mathrm{~mL}$, o tempo necessário para o retorno do sinal à linha base é maior e, consequentemente, a freqüência analítica é menor. O volume de $100 \mathrm{~mL}$ foi adotado para a amostra e o redutor.

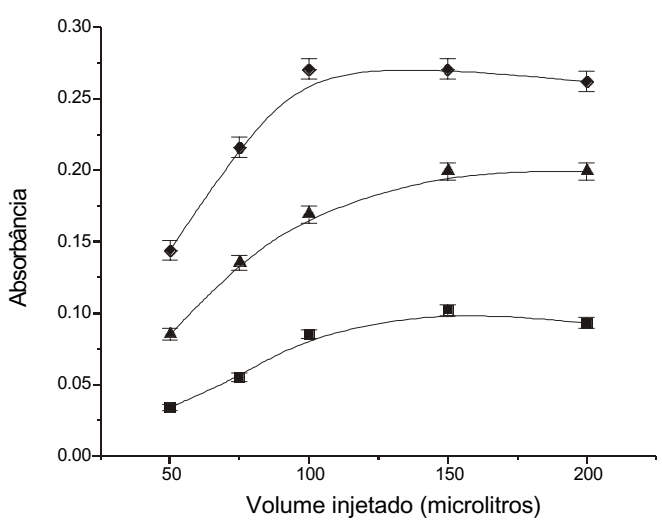

Figura 3. Efeito do volume injetado de amostra e de $\mathrm{NaBH}_{4}$ na determinação de selênio.

Condições experimentais: concentração de $\mathrm{NaBH}_{4}$ : 1,0 \% (m/ v), concentração de $\mathrm{HCl}: 1,0 \mathrm{~mol} \mathrm{~L}^{-1}$, bobina de reação: 20 $\mathrm{cm}$, vazão dos carregadores: $1,5 \mathrm{~mL} \mathrm{~min}^{-1}$, vazão de nitrogênio: $150 \mathrm{~mL} \mathrm{~min}{ }^{-1}$, temperatura de atomização: $750^{\circ} \mathrm{C}$. - - $30 \mathrm{ng}$ Se $\mathrm{mL}^{-1},-\wedge^{-} 60 \mathrm{ng}$ Se $\mathrm{mL}^{-1},-\bullet-90 \mathrm{ng}$ Se $\mathrm{mL}^{-1}$.

O efeito de diferentes concentrações de borohidreto de sódio na sensibilidade do método analítico foi verificado injetando-se volumes constantes de diferentes concentrações do redutor, no intervalo de 0,1 a 2,5 \% (m/v). A absorbância aumentou com o aumento da concentração de borohidreto de sódio até $0,5 \%$ e depois, diminuiu (Figura 4). Quando maiores concentrações de $\mathrm{NaBH}_{4}$ eram utilizadas, observou-se que além da geração do hidreto de selênio, ocorria a formação de grande quantidade de hidrogênio proveniente da reação do borohidreto de sódio com o ácido clorídrico. $\mathrm{O}$ excesso de $\mathrm{H}_{2}$ gerado provocava uma menor repetibilidade dos sinais obtidos. Desta maneira, considerou-se conveniente a utilização de uma solução de $0,5 \%(\mathrm{~m} / \mathrm{v})$ de borohidreto para a continuidade do trabalho. 


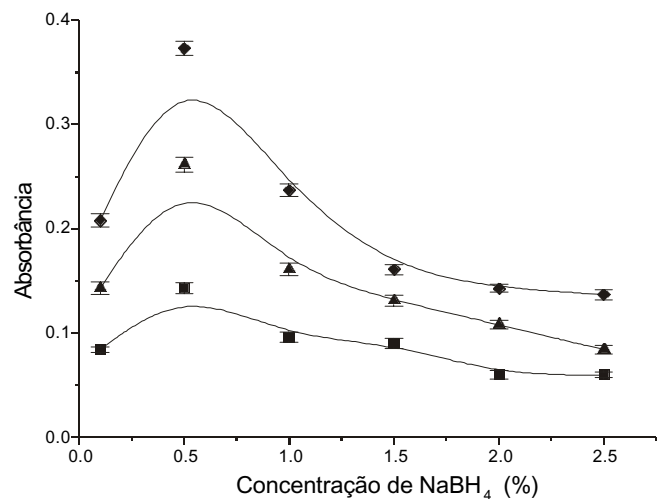

Figura 4. Efeito da concentração de $\mathrm{NaBH}_{4}$ na determinação de selênio.

Condições experimentais: concentração de $\mathrm{HCl}$ : $1,0 \mathrm{~mol} \mathrm{~L}^{-1}$, volume injetado: $100 \mathrm{~mL}$, bobina de reação: $20 \mathrm{~cm}$, vazão

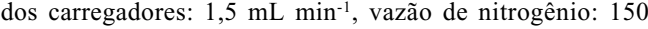
$\mathrm{mL} \min ^{-1}$, temperatura de atomização: $750^{\circ} \mathrm{C}$ - — - $30 \mathrm{ng} \mathrm{Se}$ $\mathrm{mL}^{-1},-\mathbf{\wedge}^{-} 60 \mathrm{ng} \mathrm{Se} \mathrm{mL}^{-1},-\bullet-90 \mathrm{ng} \mathrm{Se} \mathrm{mL}^{-1}$.

O efeito da variação da concentração de $\mathrm{HCl}$ no sinal analítico foi avaliado na faixa de 0,25 a 5,0 $\mathrm{mol} \mathrm{L}^{-1}$ de ácido clorídrico. Na Figura 5 observa-se um aumento de absorbância até a concentração de $\mathrm{HCl}$ de $2,0 \mathrm{~mol} \mathrm{~L}^{-1}$, permanecendo quase constante o sinal analítico para maiores concentrações de $\mathrm{HCl}$. Desde que não há diferenças significativas no sinal de absorbância para o uso de $\mathrm{HCl} 1,0 \mathrm{~mol} \mathrm{~L}^{-1}$ e 2,0 mol L-1, foi adotada a concentração de $\mathrm{HCl}$ de $1,0 \mathrm{~mol} \mathrm{~L}^{-1}$ na linha transportadora e no preparo das amostras.

O efeito da vazão das soluções transportadoras, ou seja, ácido clorídrico e água desionizada foi avaliado no intervalo de 0,5 a 2,7 mL $\mathrm{min}^{-1}$. Observou-se que a absorbância aumentou até a vazão de $1,5 \mathrm{~mL} \mathrm{~min}^{-1}$. Para vazões maiores, o sinal analítico diminuiu, pois, devido a alta vazão, a amostra e o reagente redutor não tinham tempo suficiente para reagirem antes de alcançarem o separador gás-líquido. Assim, considerou-se que a vazão de 1,5 $\mathrm{mL} \mathrm{min}^{-1}$ fornecia freqüência analítica e sensibilidade adequada aos objetivos propostos.

O efeito da vazão de nitrogênio foi estudado no intervalo de 80 a $250 \mathrm{~mL} \mathrm{~min}^{-1}$. Observouse, experimentalmente, que não havia repetibilidade no sinal analítico e a sensibilidade diminuía para

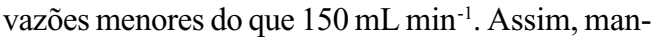

tendo o compromisso com a sensibilidade e a precisão das medidas analíticas, a vazão de $150 \mathrm{~mL}$ min $^{-1}$ foi considerada adequada para a determinação de selênio.

Para cada elemento que forma hidreto existe uma temperatura adequada. A temperatura para a atomização do hidreto de selênio foi avaliada. Observou-se que na região de abaixo de $600^{\circ} \mathrm{C}$ ocorre atomização parcial do hidreto de selênio. A máxima sensibilidade foi encontrada em $750^{\circ} \mathrm{C}$.

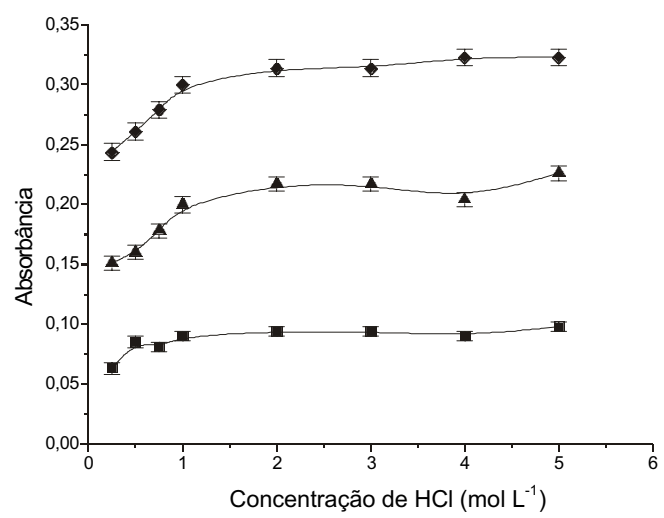

Figura 5. Efeito da concentração de $\mathrm{HCl}$ na determinação de selênio.

Condições experimentais: concentração de $\mathrm{NaBH}_{4}: 0,5 \%$ (m/ v), bobina de reação: $20 \mathrm{~cm}$, volume injetado: $100 \mathrm{~mL}$, vazão

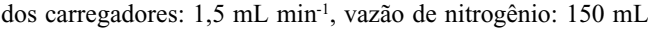
$\min ^{-1}$, temperatura de atomização: $750^{\circ} \mathrm{C}$ - - $30 \mathrm{ng} \mathrm{Se} \mathrm{mL}^{-1}$, -^-60 ng Se $\mathrm{mL}^{-1},-\bullet-90 \mathrm{ng}$ Se $\mathrm{mL}^{-1}$.

\section{Características analíticas do método proposto}

O coeficiente de correlação linear variando a concentração de selênio de 1,0 a $100,0 \mathrm{ng} \mathrm{mL}^{-1}$ é igual a 0,9955 . A precisão do método avaliada através do desvio padrão relativo (d.p.r.), calculado a partir de dez determinações em replicata, foi de $2,0 \%$ para $1,0 \mathrm{ng} \mathrm{mL}^{-1}$ de $2,3 \%$ para $100 \mathrm{ng} \mathrm{mL}^{-}$ ${ }^{1}$. O limite de detecção, considerando-se a relação sinal/ruído igual a três, segundo as recomendações da IUPAC, foi calculado em $0,3 \mathrm{ng} \mathrm{mL}^{-1}$, correspondente a $30 \mathrm{pg}$ de selênio. A freqüência analítica obtida foi de 140 determinações por hora. 
Análise de amostras de urina

A exatidão do método proposto para a determinação de selênio foi avaliada com material certificado de urina. Os resultados obtidos pelo método proposto foram comparados com os valores certificados (Tabela 1). Como pode ser observado, a determinação do selênio pelo método desenvolvido forneceu resultados satisfatórios, uma vez que foram concordantes com aqueles valores fornecidos nos certificados.

Tabela 1. Determinação de selênio $\left(\mathrm{mg} \mathrm{mL}^{-1}\right)$ em material certificado de urina SRM 2670.

\begin{tabular}{lll}
\hline Amostra & Valor certificado & Valor encontrado \\
\hline Nível normal & $0,030 \pm 0,008$ & $0,027 \pm 0,002$ \\
Nível elevado & $0,46 \pm 0,03$ & $0,45 \pm 0,008$ \\
\hline
\end{tabular}

Desde que as condições experimentais do método proposto foram avaliadas para a determinação de selênio em amostras de urina; o procedimento proposto foi aplicado a determinação de selênio em amostras de urina de pacientes submetidos a medicamentos contendo selênio. O procedimento proposto para a determinação de selênio nestas amostras foi somente o de estabelecer um método químico de análise, sem abordoar o aspecto clínico e suas conseqüências. Neste caso, procurou-se avaliar a taxa de eliminação de selênio via urina após a ingestão de medicamentos. Foi feito um estudo visando conhecer o nível de selênio na urina de pessoas que não estavam sob tratamento com dose de selênio. Para isto foi recolhida urina de três pessoas que não haviam tomado nenhum tipo de medicamento contendo selênio. Os resultados obtidos mostraram a concentração média de selênio na urina de 4,0 $\mathrm{ng} \mathrm{mL}^{-1}$. Numa amostra de urina contendo $3,5 \mathrm{ng} \mathrm{mL}^{-1}$ de selênio foram adicionadas quantidades conhecidas de selênio, a saber $1,0 \mathrm{ng} \mathrm{mL}^{-1}$ e $10,0 \mathrm{ng} \mathrm{mL}^{-1}$. Os resultados indicados na Tabela 2, mostraram a recuperação maior que $90 \%$ para a determinação de selênio.

Tabela 2. Determinação e recuperação de selênio em amostras de urina.

\begin{tabular}{llll}
\hline Amostra & Conc. adic. de Se $\left(\mathrm{ng} \mathrm{mL}^{-1}\right)$ & Conc. enc. de Se $\left(\mathrm{ng} \mathrm{mL}^{-1}\right)$ & Recuperação \\
\hline 1 & 0,0 & $3,5 \pm 0,5$ & - \\
2 & 1,0 & $0,9 \pm 0,2$ & $90,0 \%$ \\
3 & 10,0 & $10,3 \pm 0,6$ & $103,0 \%$ \\
\hline
\end{tabular}


O estudo para avaliar o perfil da eliminação do selênio na urina foi feito para uma pessoa submetida a uma dose única de medicamento e o monitoramento de selênio nas amostras de urina foi feito num período de 72 horas. A Figura 6 mostra o perfil da eliminação do selênio pela urina em função do tempo, onde o ponto correspondente ao tempo zero mostra a concentração de selênio medida antes da ingestão do medicamento.

A Figura 6 mostra uma maior eliminação do selênio nas primeiras 18 horas após a ingestão do medicamento. Após o tempo de 72 horas, concentração de selênio na urina foi de $5,5 \mathrm{ng} \mathrm{mL}^{-1}$ aproximando-se ao nível de selênio determinado na urina em condições normais.

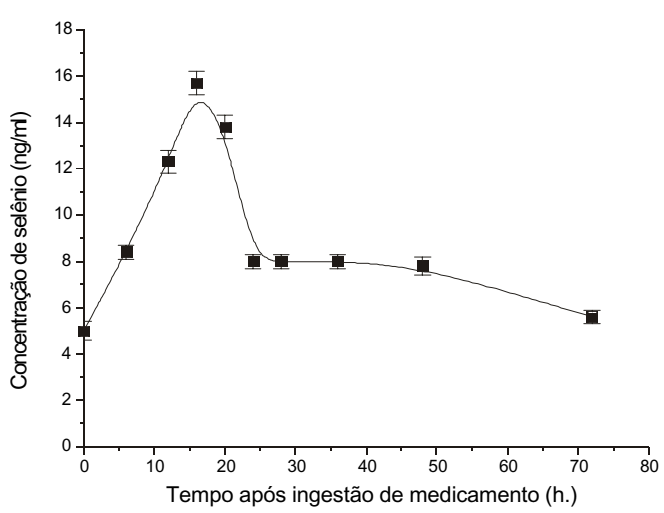

Figura 6. Monitoramento de selênio na urina de paciente submetido a medicamentos contendo selênio.

\section{Conclusões}

O método proposto é relativamente simples, de fácil operação e de baixo custo. Os sistemas de fluxo combinados à técnica de geração de hidretos são bem versáteis e proporcionam a determinação de selênio de um grande número de amostras num tempo relativamente curto. $\mathrm{O}$ sistema de fluxo por zonas coalescentes, ora proposto, apresenta vantagens como o menor consumo de $\mathrm{NaBH}_{4}\left(0,5 \mathrm{mg}\right.$ de $\mathrm{NaBH}_{4}$ para cada determinação, ou seja, $70 \mathrm{mg}$ de $\mathrm{NaBH}_{4}$ por hora) e o resíduo químico gerado no processo reacional é de $170 \mathrm{~mL} \mathrm{~h}^{-1}$, portanto, sendo economicamente interessante além de minimizar os resíduos químicos gerados no laboratório. Considerando-se os resultados obtidos, a proposta de trabalho mostrou-se potencialmente útil à determinação de selênio em amostras de urina. Assim, o procedimento desenvolvido consiste de uma alternativa viável ao monitoramento de selênio.

\section{Agradecimentos}

N.M.M. Coelho agradece à Coordenação de Aperfeiçoamento de Pessoal de Nível Superior pela bolsa de doutorado concedida.

Recebido em: 08/04/03

Aceito em: 07/07/03

N. M. M. Coelho, N. Baccan. Ultra trace determination of selenium in urine by flow injection hydride generation atomic absorption spectrometry

Abstract: Hydride generation atomic absorption spectrometry using a flow injection system by merging zones has been used successfully to determine selenium at the ultra trace level.

The detection limit was $0.3 \mathrm{ng} \mathrm{mL} \mathrm{m}^{-1}$, corresponding to $30 \mathrm{pg}$ of selenium and sample throughput of 140 samples per hour was obtained. The precision (r.s.d.) for determination of $1.0 \mathrm{ng} \mathrm{mL}-1$ of selenium is $2.0 \%$. Reagent consumption $\left(0.5 \mathrm{mg}\right.$ of $\mathrm{NaBH}_{4}$ per determination) and waste generation is low. The application of the developed methodology to the analysis of urine samples evidenced the presence of 4.0 $\mathrm{ng} \mathrm{mL} \mathrm{m}^{-1}$ of selenium for patients without administration of drugs containing selenium. For patients with administration of drugs containing selenium, the selenium concentration in the analyzed urine samples was $5.0 \mathrm{ng} \mathrm{mL}^{-1}$ until $16.0 \mathrm{ng} \mathrm{mL}^{-1}$ level. Recovery of selenium from two samples ranged from 90.0 to $103.0 \%$. The method was validated by analyzing with the standard reference material SRM 2670 urine (certified value: $30.0 \pm 8.0 \mathrm{ng} \mathrm{mL}^{-1}$ and obtained value: $27.0 \pm 2.0 \mathrm{ng} \mathrm{mL}^{-1}$ ).

Keywords: selenium, ultra trace, flow injection, hydride generation, urine 


\section{Referências}

[1] M. S. Alaejos, C. D Romero, E. M. Rodríguez-Rodríguez, Clin Chim. Acta 231 (1994) 39.

[2] H. Bergamin Filho, J. X. Medeiros, B. F. Reis, E. A. G. Zagatto, Anal. Chim. Acta 101 (1978) 9.

[3] S. Cadore, N. Baccan, J. Anal. Atom. Spectrom. 12 (1997) 637. [4] N. M. M. Coelho, A. C. Silva, C. M. Silva, Ciênc. Tecnol. 10 (2001) 109.

[5] N. M. M. Coelho, A. C. Silva, C. M. Silva, Anal. Chim. Acta 460 (2002) 227.

[6] N. M. M. Coelho, C. Parrilla, M. L. Cervera, A. Pastor, M. De La Guardia, Anal. Chim Acta 482 (2003) 73.
[7] A. K. Das, M. De La Guardia, M. L.,Cervera, Talanta 55 (2001) 1.

[8] B. Gammelgaard, K. D. Jessen, F. H. Kristensen, O. Jons, Anal. Chim. Acta 404 (2000) 47.

[9] K. Johansson, X. Luo, A. Olin, Talanta 42 (1995) 1979.

[10] L. Marin, J. Lthomme, J. Carignan, Talanta 2003, In press. [11] W. Mertz, Trace Elements in Human and Nutrition, Academic Press, New York, 5 th edn. 1986.

[12] M. L. Ruíz-Navarro, M. Navarro-Alarcón, H. L. G. De La Serrana, V. Pérez-Valero, M. C. López-Martinez, Sci. Total Environ. 216 (1998) 55.

[13] R. Sabé, R. Rubio, L. García-Beltrán, Anal. Chim. Acta 436 (2001) 215 . 